\title{
Use of e-Telemedicine for Visualization, Examination Control and Images Diagnostic in DI- COM
}

http://dx.doi.org/10.3991/ijes.v2i1.3676

Antônio Pereira Cândido, Instituto Federal de Santa Catarina, Florianópolis-SC, Brasil

Tamara Goularte Costa, Instituto Federal de Santa Catarina, Florianópolis-SC, Brasil

Rafael Andrade, Instituto Federal Catarinense, Ibirama-SC, Brasil

Abstract - This study presents the use of a telemedicine system on academic community of the Federal Institute of Santa Catarina (IFSC). The objective of this research is the evaluation of a technology solution used by the IFSC, validated by the Federal University of Santa Catarina (UFSC). This approach acquires images in DICOM format and sends it to a central server. The telemedicine system will be implemented in the IFSC and used by teachers and students to evaluate the model in real situations. For the development of this research we acquired images in DICOM format, anonymised and we sent to a local server. In another moment the local server sent the images to a central telemedicine bridge. The server converts the images to JPEG format to be viewed on our system. As a result, we provide an online database with several examinations that can be viewed by the entire IFSC academic community.

Index Terms-DICOM, PACS, Radiology, Telemedicine.

\section{Uso da telemedicina para visualização, controle de exames e diagnósticos de imagens em formato DICOM}

\author{
Antônio Pereira Cândido ${ }^{1}$, Tamara Goularte Costa ${ }^{1}$ and Rafael Andrade ${ }^{2}$ \\ ${ }^{1}$ Instituto Federal de Santa Catarina, Florianópolis, Brasil \\ ${ }^{2}$ Instituto Federal Catarinense, Ibirama, Brasil
}

\begin{abstract}
Resumo-Este estudo apresenta a utilização de um sistema de telemedicina dentro da comunidade acadêmica do Instituto Federal de Santa Catarina (IFSC). O objetivo desta pesquisa é a avaliação de uma solução tecnológica utilizada pelo IFSC, já validada pela Universidade Federal de Santa Catarina (UFSC), que adquire imagens no formato DICOM e envia para um servidor central. O sistema de telemedicina no IFSC será utilizado pelos professores e alunos para avaliação do modelo em situações reais. Para o desenvolvimento desta pesquisa, foram adquiridas imagens no formato DICOM, estas foram anonimizadas e enviadas para um servidor local e deste servidor local são enviadas para uma bridge central da telemedicina, onde são convertidas para o formato JPEG para serem visualizadas no sistema utilizado. Como resultado, tem-se a disposição um banco de dados com diversos exames que podem ser visualizados on-line por toda a comunidade acadêmica do IFSC.
\end{abstract}

Palavras-chave-DICOM, PACS, Radiologia, Telemedicina.

\section{INTRODUÇÃO}

No inicio do século XX já se podia observar a união da medicina com as tecnologias da comunicação, definindo o conceito de telemedicina, que de maneira simplificada pode ser dita como uma integração do uso da medicina com as tecnologias da informação. A telemedicina evolui cada vez mais depressa, e está em grande ascensão no que diz respeito ao diagnóstico médico e envolve diversos conceitos, padrões e sistemas, como o PACS e o DICOM [1].

O PACS é um sistema de arquivamento e comunicação de imagens voltado para o diagnóstico por imagem, que permite o pronto acesso em qualquer setor do hospital ou clínica, integra imagens e informações do paciente, como nome, endereço, sexo, dentre outros. É caracterizado por 4 sistemas: aquisição, exibição, disponibilização e armazenamento de imagens, que são distribuídas no formato DICOM [1], [2].

DICOM é o padrão global para transferência, comunicação e armazenamento de imagens médicas e informações associadas [2].

Devido ao grande crescimento desta tecnologia, há a necessidade de capacitar novos profissionais à utilização dos serviços disponibilizados pela rede. Por isso, pretende-se criar e implantar um sistema PACS no Instituto Federal de Santa Catarina (IFSC), extensão do modelo desenvolvido pela Universidade Federal de Santa Catarina (UFSC), para ser utilizado pelos professores e alunos dos cursos de radiologia e informática.

\section{Objetivos}

O objetivo geral desta pesquisa é a avaliação de uma solução tecnológica operacional de telemedicina desenvolvida e validada pela UFSC, que adquire imagens no formato DICOM e envia para um servidor central. Dentro do contexto deste trabalho, será implantada uma nova 
instância do Sistema Catarinense de telemedicina (STT/SC) no IFSC, para que os alunos e professores dos cursos de Informática e Radiologia possam utilizar a tecnologia desenvolvida pela UFSC, avaliar o modelo aprovado pela comunidade médica e selecionar soluções que possam ser exploradas academicamente pelos cursos envolvidos no projeto.

Os objetivos específicos são: capacitação dos pesquisadores, dominar as tecnologias utilizada pela UFSC e expandir o seu uso no IFSC, integrar os cursos envolvidos no projeto, ampliar o conhecimento acerca de telemedicina e disponibilizar o STT/SC para os cursos do IFSC.

Para melhor entendimento do projeto, faz-se necessário entender o que é a telemedicina e como ela interage na sociedade do estado de Santa Catarina.

\section{Telemedicina e e-telemedicina}

A organização mundial de saúde (OMS) define telemedicina como:

"A oferta de serviços ligados aos cuidados com a saúde, nos casos em que a distância é um fator crítico; tais serviços são prestados por profissionais da área da saúde, usando tecnologias de informação e de comunicação para o intercâmbio de informações válidas para diagnósticos, prevenção e tratamento de doenças e a contínua educação de prestadores de serviços em saúde, assim como para fins de pesquisas e avaliações. [3].

Dentro deste contexto, pode-se dizer que em 1910, na Inglaterra, ocorreu a primeira iniciativa para a criação e desenvolvimento da telemedicina, com a utilização do primeiro estetoscópio elétrico que funcionava por telefone. Mas apenas em 1950 a primeira imagem radiológica foi transmitida entre duas cidades [4].

No Brasil, as primeiras utilizações no âmbito da telemedicina foram registradas em 1942 com a utilização de códigos para solicitação de alguns medicamentos via telégrafo, um caso curioso, já que cada medicamento continha um código de até cinco letras, que podiam ser encomendados diretamente do Rio de Janeiro para todo o território brasileiro [5].

Em Santa Catarina, a telemedicina, no contexto utilizado pela UFSC, nasceu em 2005, com o objetivo de facilitar o acesso do cidadão aos seus exames. Usando a telemedicina, pela internet, médicos podem acessar ou enviar exames e emitir laudos à distância. Esta tecnologia foi desenvolvida pelo Grupo Cyclops do Departamento de Informática e Estatística, do Centro Tecnológico (CTC) da Universidade Federal de Santa Catarina (UFSC) e está sendo utilizado por todas as cidades do estado de Santa Catarina [6]. Ao todo são mais de 50.000 exames enviados todo mês para um servidor central e mais de 6.000 profissionais acessam exames e diagnósticos [7]. Assim nasceu a e-telemedicina: a união de um sistema web centralizado de serviços voltados para o atendimento de pacientes na área da radiologia, com a integração de profissionais médicos e pacientes em uma mesma interface gráfica [8]. A Figura 1 mostra um exemplo de uma imagem de tomografia computadorizada sedo visualizada em um navegador de internet. Nesta interface é possível efetuar medições nas imagens, mudar o janelamento, inserir textos, figuras/áreas de interesse, etc. Também é possível mudar a quantidade de imagens visíveis em cada tela clicando em botões específicos.
O sistema de telemedicina catarinense fechou o ano de 2012 com 100\% das cidades do estado de Santa Catarina tendo a presença do sistema de telemedicina, ou seja, os 295 municípios de Santa Catarina já participam do sistema catarinense de telemedicina (STT/SC). Atualmente destes 295 municípios que utilizam o sistema de telemedicina 193 já tem disponibilidade de enviar exames a distância [9] e os outros 102 municípios participam da rede de alguma forma, seja acessando exames, seja participando de palestras de capacitação a distância.

No início de 2013 o sistema catarinense de telemedicina já contava com um volume de 2,6 milhões de exames divididos em diferentes modalidades de exames, como pode ser visualizado na Tabela I.

\section{3. $P A C S$}

O sistema de armazenamento e comunicação de imagens (Picture archivingand communication system), mais conhecido como PACS, foi um dos grandes impactos na

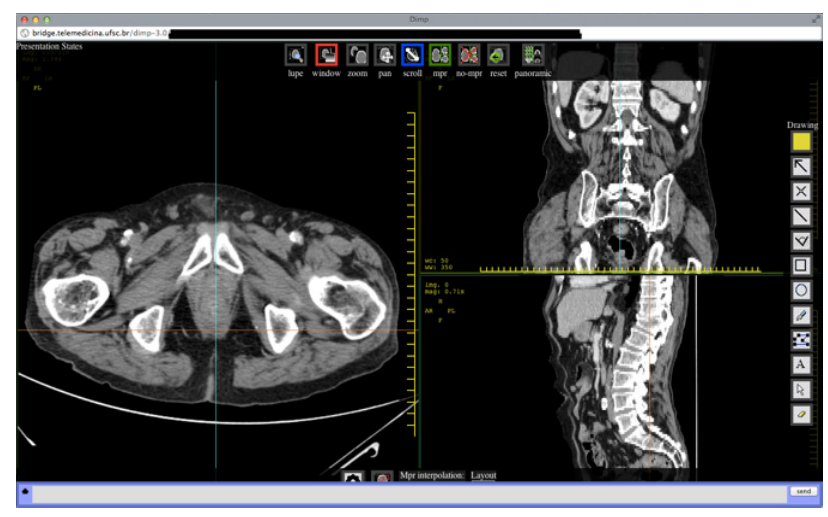

Figura 1. Visualização de uma imagem de TC diretamente na web [7].

TABELA I.

RELAÇÃO DE EXAMES E A QUANTIDADE DE EXAMES ARMAZENADOS (FONTE: ANDRADE, R. [7]).

\begin{tabular}{|l|l|r|r|}
\hline \multicolumn{3}{|c|}{ Examespormodalidades no STT } \\
\hline Abrev. & \multicolumn{1}{|c|}{ Qtde } & \multicolumn{1}{c|}{$\%$} \\
\hline AC & AnálisesClínicas & 869.765 & $41,052 \%$ \\
\hline RC & RadiologiaComputadorizada & 576.562 & $27,213 \%$ \\
\hline ECG & Eletrocardiografia & 458.389 & $21,635 \%$ \\
\hline TC & TomografiaComputadoriza- & 141.766 & $6,691 \%$ \\
\hline US & Ultrassonografia & 49.387 & $2,331 \%$ \\
\hline EN & Endoscopia & 10131 & $0,478 \%$ \\
\hline NP & Nutrição Parenteral & 3.700 & $0,175 \%$ \\
\hline RM & RessonânciaMagnética & 2819 & $0,133 \%$ \\
\hline CIN & Cintilografia & 2.806 & $0,132 \%$ \\
\hline HEM & Hemodinâmica & 1.130 & $0,053 \%$ \\
\hline COL & Colonoscopia & 987 & $0,047 \%$ \\
\hline PB & PatologiaBucal & 756 & $0,036 \%$ \\
\hline DER & Dermatoscopia & 284 & $0,013 \%$ \\
\hline OTO & Otorrinolaringologia & 126 & $0,006 \%$ \\
\hline DO & DensitometriaÓssea & 51 & $0,002 \%$ \\
\hline BRO & Broncoscopia & 33 & $0,002 \%$ \\
\hline VE & Vídeo- Eletroencefalografia & $0,001 \%$ \\
\hline Total & & $\mathbf{2 . 1 1 8 . 7 0 9}$ & $\mathbf{1 0 0 \%}$ \\
\hline
\end{tabular}


implementação de imagens médicas na telemedicina. Ele é simplesmente um sistema de arquivamento e comunicação das imagens médicas em formato digital, bastante utilizado em clínicas e hospitais, e é caracterizado por quatro subsistemas: aquisição, exibição, disponibilização e armazenamento de imagens [1], [10].

1) Aquisição de imagens

A aquisição da imagem, é o inicio de todo o processo que envolve a telerradiologia. Inicialmente, para fazer parte de um banco de dados da telemedicina, a imagem precisa estar de alguma forma em formato digital. Isto não quer dizer que imagens convencionais, adquiridas com chassi e filme não podem ser incluídas neste sistema. Por isto existem duas maneiras de adquirir este tipo de imagem:

1. O modo mais comum é a aquisição da imagem de formato digital. Esta maneira acontece simplesmente com a radiação incidindo em uma placa de fósforo, que pode estar acoplada no aparelho ou dentro de uma cassete. Em segundo momento, a imagem é transferida para um computador (worksation- onde pode ser manipulada diretamente) ou caso haja necessidade, ser impresso;

2. A outra forma de aquisição de imagens em formato digital é escanearas imagens que foram adquiridas por equipamentos convencionais. Porém, esse processo não permite a manipulação das imagens. O escaneamento, como é um processo manual, é propenso a erros e queda da qualidade da imagem [1], [10].

\section{2) Disponibilização da imagem}

A disponibilização das imagens, refere-se a distribuição das imagens de um local para o outro. Por muito tempo, enfrentaram-se problemas referentes a isto, pois não havia uma padronização das imagens. A necessidade foi tão grande, que então foi criado o padrão DICOM (Digital Imagingand Communication in Medicine), que nada mais é do que um formato para as imagens médicas, que atualmente é utilizado por todos os fabricantes de dispositivos para adquirir imagens, facilitando todo o processo [1], [2].

3) Exibição da imagem

A exibição da imagem, engloba principalmente a chamada "workstation", onde as imagens serão visualizadas para que se possa a posteriori, emitir um laudo. Esta estação de trabalho deve ser muito eficiente e ter algumas exigências que devem ser respeitadas, como possuir no mínimo dois monitores de alto-brilho e alta definição, apresentar base de dados otimizada, o tempo de espera entre a seleção da imagem e sua exibição deve ser menor ou igual a cinco segundos e o equipamento deve possuir programas básicos de processamento de imagem. Isso tudo visa garantir a qualidade e a confiabilidade no laudo médico, que exige precisão [1], [10].

\section{4) Armazenamento de imagem}

O armazenamento das imagens é o ultimo processo de um PACS e diz respeito a como as imagens serão armazenadas até serem solicitadas para visualização. Existem dois tipos de armazenamento de imagem envolvidos na telemedicina, o armazenamento rápido e o armazenamento longo. O armazenamento rápido, ou local, geralmente se faz no disco rígido do computador, onde as imagens são armazenadas em um disco magnético e permite uma recuperação rápida delas, neste caso as imagens ficam em geral uma semana armazenadas, por conta do alto custo e dos grandes volumes de dados que tem que ser armazenados no mesmo local. O armazenamento longo pode ser feito em um disco ótico, que podem ter até $10 \mathrm{~GB}$ cada disco, ou em dispositivos de armazenamento em massa, como Storages de rede. Essas informações podem ficar ali por tempo indeterminado, e em alguns casos podem ser removidas, ou enviadas para fitas magnéticas, CD-ROMs e DVDs, como backup de dados [1], [10]. A figura 2 dá uma ideia do modelo de funcionamento de um PACS distribuído em funcionamento na Rede Catarinense de Telemedicina desenvolvido pela UFSC.

$\mathrm{O}$ quadro I da figura 2 representa os servidores de armazenamento e comunicação de dados (Dicom Server e CHIS Server). O quadro II estão representados os visualizadores DICOM e workstations portáteis, que acessam os servidores representados em I. A aquisição das imagens é feita nos hospitais em diferentes momentos e são representados pelos quadros III e IV [11].

\section{D. DICOM}

Segundo a NEMA (American NationalAssociationof Eletric Machines) o DICOM (Digital Imagingand Communications in Medicine) pode ser definido como:

"padrão internacional para imagens médicas e informações relacionadas. Ele define os formatos de imagens médicas que podem ser trocados com os dados e qualidades necessárias para o uso clínico. O DICOM é implementado em quase todas as imagens radiológicas da cardiologia e dos dispositivos de radioterapia (raio- $X$, tomografia computadorizada, ressonância magnética, ultrassom, etc), e cada vez mais dispositivos em outros domínios médicos, como oftalmologia e odontologia. Com dezenas de milhares de dispositivos de imagem em uso, o DICOM é um dos mais utilizados padrões de imagens médicas do mundo. Desde a sua primeira publicação em 1993, o DICOM revolucionou a prática da radiologia, permitindo a substituição de filme radiológico com um fluxo de trabalho totalmente digital. Assim como a Internet se tornou a plataforma para aplicações de informações novas de consumo, DICOM permitiu aplicações avançadas de imagiologia médica que 'mudaram a visão da medicina clínica'. Do departamento de emergência, a testes de esforço cardíaco, para a detecção do câncer de mama, DICOM é o padrão que facilita o trabalho de imagem médica - para os médicos e para os pacientes." [12].

Ou seja, o DICOM é um padrão global para a transferência de imagens radiológicas e outras informações médicas entre computadores e é utilizado em grande escala pela rede catarinense de Telemedicina[2].

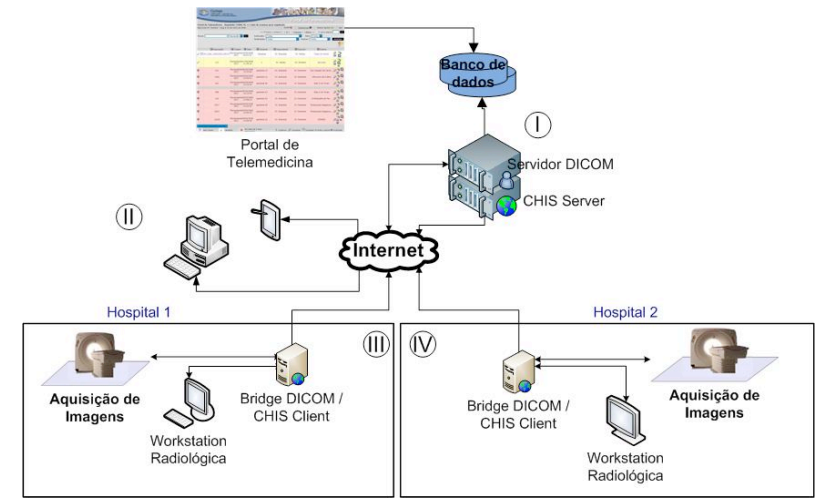

Figura 2. Modelo de funcionamento do PACS na UFSC[11] 


\section{Metodologia}

Para o desenvolvimento dessa pesquisa, foram adquiridas imagens no formato DICOM, com o intuito de formar um banco de dados, o que era essencial para o andamento do projeto.

Para que as imagens pudessem ser doadas por pacientes reais em atendimento das clinicas e hospitais de Florianópolis, foi encaminhado um projeto de pesquisa para o comitê de ética da UFSC. Os pacientes que quiserem doar seus exames para fins de estudo devem assinar um termo de consentimento e doar as imagens em formato DICOM para o IFSC. Os participantes foram avisados que a pesquisa não oferece qualquer risco a seres humanos. Também foi colocado para os pacientes que e pesquisa possui natureza educacionale não se trata de estudo experimental que venha a colocar em prática qualquer nova invenção ou procedimento pedagógico. A pesquisa foi orientada e atendida pela Resolução $n^{\circ}$ 196/96 do Conselho Nacional de Saúde, que trata dos cuidados éticos, considerando o respeito ao sujeito e à Instituição participante de todo o processo.

Cada participante recebeu um termo, onde foi explicado o objetivo da pesquisa e solicitado que o paciente assinasse o termo, caso ele autorizasse a doação das imagens.

O segundo passo da pesquisa foi disponibilizar a arquitetura de software desenvolvida pela UFSC no IFSC. Esta tecnologia consiste em um modelo de telemedicina diagnóstica assíncrona, que nada mais é um conjunto de ferramentas de software para acesso aos dados, imagens e sinais médicos, elaboração de laudos e registro do histórico básico de procedimentos. Esta solução permite aos médicos e outros profissionais de saúde acessar e inserir informações sobre paciente (dados demográfico, resumo clínico, exames e procedimentos) de forma rápida e em larga escala (larga abrangência geográfica e grande número de procedimentos). Cada nova notação e exame são visualizados na forma de uma "nova mensagem" e o trabalho de geração do laudo e regulação de solicitações é bastante simplificado. O gerenciamento é controlado pela associação de papéis de acesso a cada usuário, sendo que tais papéis possuem acessibilidade pré-definida pelo gestor institucional do serviço de saúde. Assim, através de um portal WEB integrado (STT/SC), a grande maioria dos procedimentos de acesso e gerenciamento a distância de informações em saúde pode ser realizado de forma assíncrona, em larga escala, pelos profissionais de saúde, pelos gestores e pelos pacientes. Destaca-se que serviço é destinado a atender grande volume de exames, em geral, atende a triagem para separação dos casos normais (cerca de $80 \%$ ) dos exames com anormalidades e, posteriormente, encaminhamento dos achados conforme nível assistencial.

Para que o sistema funcionasse em uma nova instância fora do contexto hospitalar, foi preparada uma nova base de dados para diferenciar os exames de estudos, dos exames de atendimentos reais. Foi instalada e utilizada uma base de dados de exames em conjunto com um servidor DICOM desenvolvido pela UFSC. Este servidor recebe as imagens em um arquivo DICOM formado por um cabeçalho padrão e um conjunto de dados. O cabeçalho possui, entre outros dados, informações sensíveis, como o nome do paciente e da instituição onde o exame foi realizado. Por motivos éticos, foi necessária a anonimização destas informações para que elas pudessem ser propriamente utilizadas.
A razão de ser alterado também o nome da instituição é mais de caráter técnico do que ético, e está relacionado com o modo como o servidor DICOM local se comunica com o servidor DICOM do STT/SC.

Quando o servidor do STT/SC recebe as imagens DICOM pela internet, é verificado no cabeçalho dos arquivos a palavra-chave referente a instituição e estes arquivos são organizados de acordo com sua procedência. Como os exames do banco de dados foram doados por diversas clínicas, instituições e pacientes, foi modificado o conteúdo da tag "InstitutionName" para "Instituto Federal de Santa Catarina" para que, ao chegarem no servidor, sejam organizados corretamente.

A tarefa de anonimizar os dados de pacientes e alterar o nome da instituição para que possam ser visualizadas no servidor DICOM do STT/SC, deve ser feita imagem por imagem. Em um primeiro momento, este procedimento foi feito manualmente, pois não saberia se a alteração dos dados diretamente na imagem poderia ou não corromper o arquivo DICOM e inutilizar o exame do paciente. Por este motivo, vários testes foram efetuados antes de se automatizar o processo.

Devido ao grande volume de arquivos (um único exame pode conter centenas imagens) e devido ao grande número de exames doados ao IFSC, foi necessário que este processo fosse automatizado. Utilizando o DCMTK[13], uma coleção de bibliotecas e aplicações para manipular arquivos DICOM, foi elaborado um algoritmo de programação no terminal do sistema operacional Linux, que procura os arquivos pela sua extensão e realiza as alterações automaticamente, independente do número de imagens e exames (DCMTK - DICOM Toolkit). Para que este arquivo de script possa ser executado, basta que sejam colocadas os exames em formato DICOM na pasta "/DICOM DATA" do servidor local do IFSC. Este servidor fora chamado de Bridge DICOM Local.

A Bridge Local é responsável pelo armazenamento temporário e o envio das imagens recebidas para o DICOM Server. A Bridge é encarregada pela centralização e armazenamento dos exames realizados em todas as instituições interligadas a rede catarinense de Telemedicina. Em cada Bridge existe uma ferramenta chamada de CHIS (Cyclops HealthcareIntegration System), que é responsável pela administração e manutenção das imagens.

Deste modo, as imagens que se encontram na estação local (IFSC) e que estão em uma pasta DICOM DATA, são enviadas através da internet para uma Bridge Central (Servidor DICOM) da telemedicina, onde são convertidas para o formato JPEG, para serem mostradas no sistema web da telemedicina. A Figura 3 mostra um exemplo de visualização um exame de Tomográfica Computadorizada da face de um paciente anonimizado cadastrado no STT.

Com este processo concluído, tem-se à disposição da instituição um banco de dados com diversos exames, de diferentes modalidades, que podem ser visualizados pelo curso de radiologia como material de pesquisa e estudos. Por este sistema, os alunos podem aprender a utilizar o sistema que é disponibilizado para unidades de saúde do estado de Santa Catarina. Nele, os alunos podem aprender a cadastrar dados de pacientes, dados de atendimento, como indicação clinica, fatores de risco, possíveis hipóteses diagnósticas, etc. Podem também enviar imagens de exames/estudos de pacientes, visualizar os exames com e sem laudo. Aprenderem a emitir laudos, gerar protocolos 
de acesso ao sistema, e encaminhar os exames para os médicos que requisitam exames no STT/SC. Isso pode ser feito usando um ambiente controlado como simulação de atendimentos das unidades de saúde do estado de Santa Catarina. Desta forma, os alunos já podem, dentro da academia, aprenderem a usar/manipular sistemas de uso em ampla escala.

\section{RESUlTADOS E DiscUSSÕES}

O intuito desta pesquisa é a utilização do sistema de telemedicina pelas unidades curriculares dos cursos do IFSC. As unidades curriculares mais beneficiadas com os resultados deste projeto são informática em saúde, técnicas radiológicas, tomografia computadorizada, radiologia digital e ressonância magnética do Curso Superior de Tecnologia em Radiologia. Os alunos podem aprender a utilizar mais uma ferramenta de telemedicina, visualizar imagens médicas correlacionando com a prática. Já no Curso Superior de Tecnologia em Gestão da Tecnologia da Informação as unidades curriculares beneficiadas são as de lógica de programação e sistema operacional Linux, onde os alunos podem pôr em prática as técnicas aprendidas em um ambiente computacional. Como este projeto ainda está em andamento, os resultados estão surgindo aos poucos, e não é possível mensurar esses resultados. Muitos exames já foram, e diversos outros estão sendo enviados para o servidor local, onde são anonimizadas as informações pessoais dos pacientes, e após serão enviado para a bridge central e será disponibilizada para acesso à comunidade acadêmica do IFSC. Para ampliar o banco de dados, foi enviado um projeto aperfeiçoado, para o comitê de ética da UFSC, embasado no que o objetivo da pesquisa é compor um banco de exames de imagem com dados reais e que serão doados pela população de Santa Catarina.

Além disso, foi preparado um treinamento do sistema e um questionário baseado em [14], para os alunos e professores do IFSC, eles utilizarão o sistema por alguns meses e em seguida os discentes e docentes do IFSC responderão o questionário. Este questionário permitirá entender melhor a aplicabilidade do modelo do sistema por parte da comunidade acadêmica.

Em um primeiro momento já se tem grandes avanços, principalmente quando se fala na anonimização das imagens. As primeiras anonimizações foram feitas individualmente imagem por imagem, e logo, foi desenvolvido um aprendizado mais profundo, aperfeiçoando as técnicas de anonimização, executando este processo agora automaticamente com um grupo de imagens muito maior.

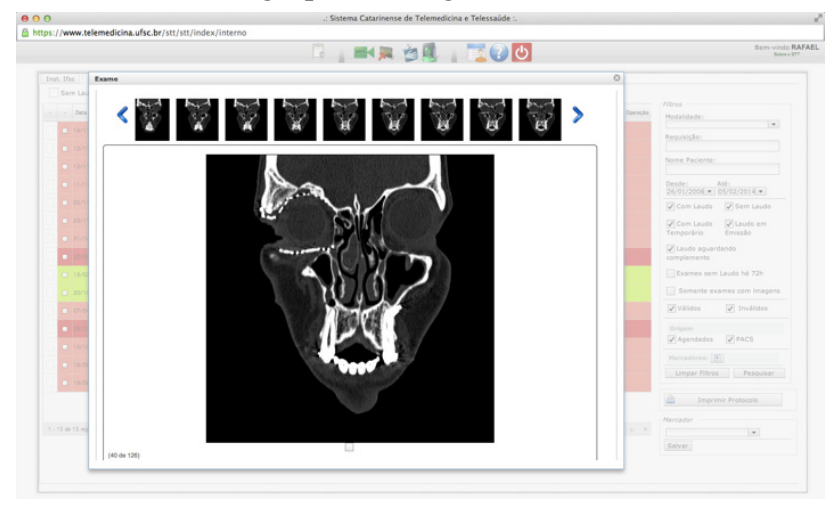

Figura 3. Exemplo de um exame de TC visualizado no STT.
Pode-se concluir que o principal resultado obtido deste projeto foi ampliação da rede de Telemedicina, aliado a novos utilizadores que foram capacitados a usarem o sistema. Este modelo promove uma melhora significativa no atendimento ao paciente, torna mais rápido o diagnóstico e gera um histórico de informações do paciente, impulsionando uma melhor qualidade de vida dos pacientes que fazem uso da telemedicina em Santa Catarina.

\section{REFERÊNCIAS}

[1] AZEVEDO-MARQUES, P. M. Et Al. "Implantação de um miniPACS (Sistema de Arquivamento e Distribuição de Imagens) em Hospital Universitário”. Radiol. Bras. 2001. Vol. 34. p. 221-224.

[2] AZEVEDO-MARQUES, P. M.; SALOMÃO, S. C. "PACS: Sistema de Arquivamento e Distribuição de Imagens". Revista Brasileira de Física Médica, 2009. Vol. 3. p. 131-139.

[3] Organização Mundial de Saúde. "Telemedicine: opportunitiesanddevelopments in MemberStates". 2009. Disponível em $<$ http://www.who.int/goe/publications/goe_telemedicine_2010.pdf $>$ Acesso em 11 de jul. 2013.

[4] Gomes, André Guanaes; Moraes Jr., João Batista Moura Xavier de;Timerman, Sergio. "Telemedicina e sua aplicação em emergências".Disponível em: http://www.projetotime.com/apostilas/Cap.08.pdf. Acesso em 02 de Set. 2013.

[5] KHOURI, S.G.E. "Telemedicina: análise da sua evolução no Brasil”. Dissertação de Mestrado. São Paulo, 2003.Disponível em: http://www.teses.usp.br/teses/disponiveis/5/5160/tde-24102007143128/pt-br.php. Acesso em: 02 de set. 2013.

[6] ANDRADE, R. ; MACEDO, D. D. J. ; WALLAUER, J. ; WANGENHEIM, Aldo Von." Building a NationalTelemedicine Network". IT Professional, v. 10, p. 12-17, 2008.

[7] ANDRADE, R. ; WAGNER, H. M. ; WANGENHEIM, A. V. "Telemedicina em Santa Catarina, um projeto Sustentável". CBIS'2012.

[8] STT/SC Sistema Catarinense de Telemedicina e Telessaúde. Disponível em <https://www.telemedicina.ufsc.br/rctm/.> Acesso em 01 de Outubro de 2012.

[9] WANGENHEIM A. V., et. al. "Implementing DICOM StructuredReporting in a Large-ScaleTelemedicine Network". Telemedicineand e-Health. July 2013, 19(7): 535-541. http://dx.doi.org/10.1089/tmj.2012.0103

[10] SILVA, C. D. S. Da; SENA, L. M. C. "Diretrizes para a Implantação de um Sistema PACS - Picture Archivingand Communication System". INATEL - Instituto Nacional de telecomunicações, 2008.

[11] Martini, Richard A. S.; Macedo, Douglas D. J.; Andrade,Rafael; Wangenheim' Aldo v. "Um Modelo de Gerenciamento Remoto de PACS Geograficamente Distribuído". Congresso Brasileiro de Informática em Saúde - CBIS, 2008. Disponível em: http://www.sbis.org.br/cbis11/arquivos/722.pdf. Acesso em: 05 de fev. 2014.

[12] AMERICAN NATIONAL ASSOCIATION OF ELETRIC MACHINES. About DICOM. Disponível em $<$ http://medical.nema.org/Dicom/about-DICOM.html $>$ Acesso em: 29 de nov. 2012

[13] DICOM Toolkit - DCMTK. Disponível em: http://dicom.offis.de/dcmtk.php.en. Acesso em: 05 de fev. 2014.

[14] WANGENHEIM, A. von; NOBRE, L.F.S.; TOGNOLI, H.; NASSAR, S.M.; HO, K. "UserSatisfactionwithAsynchronousTelemedicine A StudyofUsersof Santa Catarina's System ofTelemedicineand Telehealth". TelemedicineJournaland e-Health, v. 18, p. 339-346, 2012.

\section{AUTORES}

Antônio Pereira Cândicoé doutor em Engenharia de Produção pela Universidade Federal de Santa Catarina e Coordenador do Curso Superior de Tecnologia de Gestão da Tecnologia da Informação do Instituto Federal de Santa Catarina, Av. Mauro Ramos, 950, Florianópolis-SC, Brasil. (e-mail: apec@ifsc.edu.br). 
Tamara Goularte Costa é acadêmica do Curso Superior de Tecnologia em Radiologia do Instituto Federal de Santa Catarina, Av. Mauro Ramos, 950, Florianópolis-SC, Brasil. (e-mail: tamaracostaa@hotmail.com).

Rafael Andrade é doutor em Engenharia e Gestão do Conhecimento e Professor titulardo Curso Informática do Instituto Federal Catarinense, na Rua Getúlio Vargas, 3006, Ibirama-SC, Brasil (e-mail: rafael.andrade@ibirama.ifc.edu.br)

Submitted 19 March 2014. Published as re-submitted yb the authors 05 April 2014. 